\title{
Prognostic prediction model for glioblastoma: a metabolic gene signature and independent external validation
}

\section{Chuxiang Lei}

Peking Union Medical College Hospital

\section{Wenlin Chen}

Peking Union Medical College Hospital

\section{Yuekun Wang}

Peking Union Medical College Hospital

\section{Binghao Zhao}

Peking Union Medical College Hospital

\section{Penghao Liu}

Peking Union Medical College Hospital

\section{Ziren Kong}

Peking Union Medical College Hospital

\section{Delin Liu}

Peking Union Medical College Hospital

\section{Congxin Dai}

Peking Union Medical College Hospital

\section{Yaning Wang}

Peking Union Medical College Hospital

Yu Wang ( $\square$ ywang@pumch.cn )

Peking Union Medical College Hospital https://orcid.org/0000-0002-7175-4649

\section{Wenbin Ma}

Peking Union Medical College Hospital

\section{Research}

Keywords: glioblastoma, metabolic gene, prognostic model, GEO, TCGA

Posted Date: March 21st, 2020

DOI: https://doi.org/10.21203/rs.3.rs-18209/v1 
License: (c) (i) This work is licensed under a Creative Commons Attribution 4.0 International License. Read Full License 


\section{Abstract}

Background. Glioblastoma (GBM) is the most common primary malignant intracranial tumor and is closely related to metabolic alterations. However, few accepted prognostic models are currently available, especially models based on metabolic genes. Methods. Transcriptome data were obtained for all patients diagnosed with GBM from the Gene Expression Omnibus (GEO) (training cohort, $n=369$ ) and The Cancer Genome Atlas (TCGA) (validation cohort, $n=152$ ) with the following variables: age at diagnosis, sex, follow-up and overall survival (OS). Metabolic genes according to Kyoto Encyclopedia of Genes and Genomes (KEGG) pathways were filtered, and a Lasso regression model was constructed. Survival was assessed by univariate or multivariate Cox proportional hazards regression and Kaplan-Meier analysis, and we also conducted an independent external validation to examine the model.

Results. There were 341 metabolic genes that showed significant differences between normal brain tissues and GBM tissues in both the training and validation cohorts, among which 56 genes were significantly correlated with the OS of patients. Lasso regression revealed that the metabolic prognostic model was composed of 18 genes, including COX10, COMT , and GPX2, with protective effects, as well as OCRL and RRM2, with unfavorable effects. Patients classified as high-risk by the risk score from this model had markedly shorter OS than low-risk patients $(P<0.0001)$, and this significant result was also observed in the independent external validation cohort $(P<0.001)$. Conclusions. The prognosis of GBM was dramatically related to metabolic pathways, and our metabolic prognostic model had high accuracy and application value in predicting the OS of GBM patients. Background. Glioblastoma (GBM) is the most common primary malignant intracranial tumor and is closely related to metabolic alterations. However, few accepted prognostic models are currently available, especially models based on metabolic genes.

Methods . Transcriptome data were obtained for all patients diagnosed with GBM from the Gene Expression Omnibus (GEO) (training cohort, $n=369$ ) and The Cancer Genome Atlas (TCGA) (validation cohort, $n=152$ ) with the following variables: age at diagnosis, sex, follow-up and overall survival (OS). Metabolic genes according to Kyoto Encyclopedia of Genes and Genomes (KEGG) pathways were filtered, and a Lasso regression model was constructed. Survival was assessed by univariate or multivariate Cox proportional hazards regression and Kaplan-Meier analysis, and we also conducted an independent external validation to examine the model. Results. There were 341 metabolic genes that showed significant differences between normal brain tissues and GBM tissues in both the training and validation cohorts, among which 56 genes were significantly correlated with the OS of patients. Lasso regression revealed that the metabolic prognostic model was composed of 18 genes, including COX10 , COMT , and GPX2, with protective effects, as well as OCRL and RRM2, with unfavorable effects. Patients classified as high-risk by the risk score from this model had markedly shorter OS than low-risk patients $(P<0.0001)$, and this significant result was also observed in the independent external validation cohort $(P<0.001)$.

Conclusions. The prognosis of GBM was dramatically related to metabolic pathways, and our metabolic prognostic model had high accuracy and application value in predicting the OS of GBM patients. 


\section{Background}

Glioblastoma (GBM, WHO grade IV) is a heterogeneous group of primary malignant central nervous system (CNS) tumors with an incidence of 5.25 per 100,000 population per year(1). Among all the tumors in the CNS, GBM shows the most invasiveness and the highest malignancy. Although the clinical treatment of GBM includes surgery, radiotherapy, chemotherapy, targeted therapy, immune therapy and their combinations $(2,3)$, the prognosis remains unfavorable. According to the statistical results of the Chinese Glioma Collaboration Group in 2016, the median overall survival (OS) of GBM patients is only 14.4 months(4), while the five-year survival rate is less than $5 \%(5)$. Therefore, the establishment of a prognostic prediction model is vital for making effective clinical decisions and has become one of the current research hotspots.

Metabolic pathways are closely related to life processes, and the alteration of any metabolic pathway has also become a driving factor for the occurrence and progression of tumors and can serve as a novel hallmark(6). Warburg first observed that the glycolysis process was preferred to the tricarboxylic acid pathway in tumor cells, even under conditions with sufficient oxygen(7). Further studies proved that the metabolic processes of glutamate and fatty acids differed significantly in hepatocellular carcinoma (HCC) cells(8), while blocking glutamate induced divergent metabolic programs to overcome tumor immune evasion(9). In GBM, previous studies suggested that mutations of isocitrate dehydrogenase (IDH) influenced the metabolism of alpha-ketoglutarate, generated excessive 2-hydroxyglutarate and altered DNA and histone methylation. Therefore, IDH mutations mediate the abnormality of metabolic pathways and significantly influence the response to treatment and prognosis of GBM(10). In addition, other metabolic reprogramming phenomena were also detected, including the transformation in glycolysis and oxidative phosphorylation(11). In terms of prognosis, GBM patients with IDH1 mutations presented a longer OS after surgery(12). Therefore, targeting abnormal metabolic pathways has become a possible direction for GBM.

Considering the poor prognostic status of GBM patients and the absence of generally accepted predictive models, it is of great significance to establish a survival prediction model. Metabolic pathway alterations may serve as prognostic factors, and studies have suggested that hypoxic glucose metabolism is a potential factor(13). Due to the dramatic changes in the metabolic pathways in GBM, the expression levels of metabolic genes are expected to predict prognosis from a new prospective.

In the present study, the differentially expressed genes between normal brain tissues and GBM tissues were detected through database retrieval, and all the metabolic genes were extracted to construct a predictive prognostic model. Moreover, an independent external validation was performed to evaluate the efficiency of our predictive model. This study provides new insights into the prognosis of GBM patients and may provide novel tumor markers or therapeutic targets, further promoting the progress of GBM diagnosis and treatment.

\section{Materials}




\section{Study design and data collection}

This is a retrospective cohort study that included all the transcriptome and clinical data of patients from the Gene Expression Omnibus (GEO) and The Cancer Genome Atlas (TCGA) databases. The training cohort comprised patients from the GEO database, and a total of 904 series were found. To be included, the series must originate from the GBM tissue of humans and have complete transcriptome profiling by array as well as clinical information including survival state and time. Series that were obtained from other sequencing methods or cell strains, those that contained fewer than 30 patients or those that were from patients in any therapeutic clinical trials were excluded. Eventually, 3 series (GSE83300(14), GSE74187(15), and GSE13041(16)) with transcriptome and clinical data were included (Fig. 1). The only endpoint of our study was OS, and to ensure the reliability of the follow-up outcomes, we also excluded patients whose follow-up times were less than 30 days. After the above screening, a total of 369 patients met the criteria and formed the training cohort. The validation cohort consisted of patients from the TCGA database. There were 606 clinical records in total, of which 169 patients had transcriptome data. Similarly, patients followed for less than 30 days were excluded, and 152 patients constituted the validation cohort.

\section{Differential gene acquisition}

All the sequencing data in the GEO database were derived from GBM tissue; therefore, it is important to obtain the transcriptome data of normal human brain tissue for comparison. We consulted the GenotypeTissue Expression (GTEx) database, which is a tissue biobank of more than 7,000 autopsy samples from healthy human donors during their lifetime. A total of 1,152 transcriptome sequencing data points of brain tissue were used as normal controls. Before comparing the differences in gene expression between normal brain and GBM tissues, we conducted batch normalization by surrogate variable analysis (SVA) to eliminate errors caused by batch effects between different chips, which was achieved through an $\mathrm{R}$ package called "sva"(17). Normalization and log2 transformation of the transcripts were then employed for the expression profiles. There were 7,967 annotated protein-coding genes used for differential expression analyses by the "Limma" version 3.42.0 R package(18). False discovery rate $(F D R)<0.05$, which was calculated by the $P$ value obtained from the Wilcoxon test, and $\log _{2}$ fold change $\left(\log _{2} \mathrm{FC}\right)>2$ were considered statistically significant.

\section{Identification of intersecting metabolic mRNAs in GEO and TCGA}

We identified metabolic genes from the genes based on the Kyoto Encyclopedia of Genes and Genomes (KEGG) pathways. The metabolic genes were selected if 1 ) they showed consistent expression patterns in the GEO cohort and transcriptome data from GTEx and (2) they were listed in the TCGA cohort. Another differential expression analysis of intersecting metabolic genes was performed through the "Limma" version 3.42.0 R package(18). Genes with $F D R<0.05$ and $\log _{2} F C>0.5$ or $<-0.5$ were considered 
statistically significant. The metabolic genes with significantly different expression levels were used in subsequent analyses and models.

\section{Construction of the predictive prognostic model of metabolic genes}

Univariate Cox regression of metabolic genes with significantly different expression levels was performed to identify candidate genes. Then, we conducted Lasso-penalized Cox regression analysis of those candidate genes to identify the prognosis-related metabolic genes and construct the prognostic gene signature as previously mentioned(19). The prognostic gene signature is shown as

risk score $=\sum_{i=1}^{n}$ Coefficient $_{m R N A_{i}} \cdot$ expression of $m R N A_{i}$,

through which the risk score of each patient can be calculated. The patients were divided into high- and low-risk groups according to the median of the summarized risk scores. The "survival" and "survminer" $\mathrm{R}$ packages were used to compare the differences in OS between the two groups of patients and draw the Kaplan-Meier survival curves.

\section{External validation of metabolic gene prognostic model}

Using the same cut-off value from the above steps, 152 patients in the TCGA cohort were also separated into 2 groups, and similar survival analysis was also performed on this cohort to validate the accuracy of the model.

\section{Construction and validation of a predictive nomogram and Gene Set Enrichment Analysis (GSEA)}

A nomogram was built by including all independent prognostic factors, including sex, age, and risk score, in our study(20). With the bootstrap method with 1,000 resamples, we used calibration curves and the concordance index (C-index) to describe the calibration and distinction of the nomogram, respectively. The metabolic gene signature, sex, and age were compared with receiver operating characteristic (ROC) curves, and a multivariate Cox regression model including sex, age, and risk score was built to reflect the combination of risk factors. To further investigate the potential underlying KEGG pathways of the gene signature, GSEA was utilized to find enriched terms in the training and validation cohorts(21).

\section{Statistical analysis}

Independent $t$ test, the Wilcoxon test or the Mann-Whitney $\mathrm{U}$ test was performed as appropriate to evaluate differences in scale or ordinal variables. Survival was assessed using Cox proportional hazards regression and Kaplan-Meier analysis. Two-sided $P$ values less than 0.05 were considered statistically significant. All statistical analyses were conducted with SPSS software (version 24.0, IBM SPSS statistics) and R software v3.6.1 (R Foundation for Statistical Computing, Vienna, Austria). 


\section{Result}

\section{Differential gene expression between normal brain and GBM tissues}

We first compared the transcriptome profiling of human GBM and normal brain tissues. A total of 769 genes with significant differences in expression between the 2 groups were included (see Supplemental Table S1 describing the list of all the differentially expressed genes). To construct the predictive prognostic model of metabolic genes in the training cohort, we conducted KEGG pathway analysis, and 616 metabolism-related genes were extracted. After comparison, we found that these genes were all included in the validation cohort. Another differential gene analysis was conducted, and 341 metabolic genes with significant differences that appeared in both cohorts composed the candidate gene set (see Supplemental Table S2 and Figure S1).

\section{Screening for prognosis-related metabolic genes}

To explore the relationship between prognosis and metabolic genes, we conducted univariate Cox regression and revealed hazard ratios (HRs) of 56 genes that were significantly related to OS in the training cohort (see Supplemental Figure S2). Favorable genes such as COX10, COMT, and GPX2 had negative HRs, suggesting that their high expression indicated a better prognosis. In contrast, the higher expression of unfavorable genes, such as OCRL and RRM2, was prone to worse outcomes. However, the HR of each gene's expression level was extremely close to 1, indicating that the predictive power of a single gene for prognosis was limited, and a more efficient predictive model was needed.

\section{Lasso regression model and Kaplan-Meier analysis of the training and validation cohorts}

To further investigate the influence of metabolic genes on prognosis, we conducted Lasso regression. Among the 56 genes that were significantly associated with OS in univariate Cox regression, 18 genes were eventually retained in the model (Table 1). There were 11 genes with negative coefficients, including COX10, COMT, and GPX2, indicating a protective effect on prognosis. Seven genes with positive coefficients, including OCRL and RRM2, suggested a worse prognosis. According to the coefficients and expression levels of the 18 genes, the risk score of each patient was calculated. The training cohort was divided into 2 groups according to the median risk score, and survival analysis presented significant differences between the 2 groups $(p<0.0001)$ (Fig. 2A). Furthermore, we used the same median risk score of the training cohort as a threshold to stratify the validation cohort. The same conclusion was reached: patients with lower risk scores had significantly better OS than those with higher risk scores $(p<0.001)$ (Fig. 2B). 
Table 1

Genes with $\log _{2} F C$, FDR and their coefficients in the prognostic model after LASSO regression.

\begin{tabular}{|c|c|c|c|c|c|}
\hline \multirow[t]{2}{*}{ Gene symbol } & \multicolumn{2}{|c|}{ Training cohort } & \multirow[t]{2}{*}{ Coefficients $^{\dagger}$} & \multicolumn{2}{|c|}{ Validation cohort } \\
\hline & $\log _{2} \mathrm{FC}^{\#}$ & $\mathrm{FDR}^{\&}$ & & $\log _{2} \mathrm{FC}^{\#}$ & FDR $^{\&}$ \\
\hline cox10 & -0.28 & $<0.001^{*}$ & -0.006377627 & 0.18 & $0.030^{*}$ \\
\hline COMT & -0.26 & $<0.001^{\star}$ & -0.002879681 & -0.43 & $<0.001^{\star}$ \\
\hline GPX2 & -0.29 & $<0.001 *$ & -0.002808587 & 0.17 & $0.017^{\star}$ \\
\hline AKR1B10 & -0.67 & $<0.001 *$ & -0.002396644 & -0.72 & 0.3 \\
\hline GLUD1 & -0.48 & $<0.001^{\star}$ & -0.001860585 & -0.51 & $<0.001 *$ \\
\hline LPCAT3 & -0.44 & $<0.001 *$ & -0.001453787 & 0.26 & $0.016^{*}$ \\
\hline GSTM3 & -0.51 & $0.046^{\star}$ & -0.001408588 & -0.55 & $<0.001^{*}$ \\
\hline GLUD2 & -0.76 & $0.002^{\star}$ & -0.001196549 & -0.13 & 0.3 \\
\hline MTHFD2 & -0.65 & $0.011^{*}$ & -0.001020503 & -0.65 & $<0.001 *$ \\
\hline EPHX1 & -0.34 & 0.1 & -0.000394242 & -0.29 & 0.1 \\
\hline PDE8B & 0.97 & $<0.001 *$ & -0.000262491 & 0.03 & 0.4 \\
\hline GLUL & 0.15 & 0.1 & 0.000358099 & 0.08 & 0.4 \\
\hline MDH1 & 0.48 & $0.002^{*}$ & 0.000502893 & -0.19 & $<0.001^{*}$ \\
\hline GBE1 & 0.60 & $<0.001 *$ & 0.001414345 & 0.66 & $<0.001^{*}$ \\
\hline PFKL & 0.50 & $<0.001^{\star}$ & 0.002176092 & 0.44 & $<0.001^{\star}$ \\
\hline PAFAH1B1 & 0.28 & $0.003^{*}$ & 0.002265462 & 0.47 & $<0.001^{\star}$ \\
\hline RRM2 & 0.75 & $<0.001 *$ & 0.003109864 & 0.85 & $<0.001^{*}$ \\
\hline OCRL & 0.60 & $0.002^{\star}$ & 0.004181019 & 0.36 & $<0.001^{*}$ \\
\hline \multicolumn{6}{|c|}{ \# $\log _{2} \mathrm{FC}=\log _{2}$ (mean expression of high-risk group/ mean expression of low-risk group) } \\
\hline \multicolumn{6}{|c|}{ \& FDR was calculated by P value from Wilcoxon test. } \\
\hline \multicolumn{6}{|c|}{ † Coefficients were calculated by Lasso regression. } \\
\hline \multicolumn{6}{|c|}{ * marked significant differences. } \\
\hline $\log _{2} F C: \log _{2} f$ & change; FI & false disc & ry rate. & & \\
\hline
\end{tabular}




\section{Increasing risk scores suggested worse prognosis in both the training and validation cohorts}

To further examine the relationship between the expression level of the 18 genes and prognosis, we drew heatmaps (Fig. 3A\&D) that suggested that the gene expression profiles of patients in the low- and highrisk groups showed no significant difference at the single-gene level. All the patients in both the training and validation cohorts were ranked in ascending order of risk scores (Fig. 3B\&E). Compared to the training cohort, more patients in the validation cohort fell into the low-risk group. Furthermore, we plotted each patient according to their risk scores and survival time (Fig. 3C\&F). On the x-coordinate, patients were uniformly ranked in ascending order of risk scores as mentioned above, and the $y$-axis reflected the survival time of each patient. The distributions of the survival state (deceased or alive) and survival time were presented as negatively related to the risk score.

\section{Multivariate Cox regression model and ROC analysis of the training and validation cohorts}

We incorporated sex and age into the model to further explore other clinical factors related to prognosis (Fig. 4). In the training cohort, both univariate (see Supplemental Table S3) and multivariate Cox regression (Fig. 4A) indicated that age and risk score were significantly related to OS, with HRs of 1.043 and 6.383 , respectively, in the multivariate model. However, patient sex was not an independent risk factor. The same model applied to the validation cohort reached similar conclusions (Fig. 4B), suggesting that the risk score calculated by our Lasso model was an independent risk factor for GBM patients (HR 1.251 with $95 \% \mathrm{Cl} 1.019-1.534)$. ROC analysis of the training cohort indicated that the risk score had better predictive power than age (Fig. 4C), while this effect was not observed in the validation cohort (Fig. 4D). The dissimilar conclusions might be due to the difference in baseline data, such as age, between the 2 groups. It was possible that the predictive power of age improved gradually as age increased. To further optimize the model, nomograms of the 2 groups were plotted to illustrate the relationship between sex, age, and risk score and prognosis (Fig. 4E\&F).

\section{Multiple GSEA of the training and validation cohorts}

Multiple GSEA was performed, and 155 and 178 enriched KEGG pathways were found in the training and validation cohorts, respectively. There was a large number of overlapping enrichment pathways between the two groups, including a majority of metabolism-related gene sets, as expected (Fig. 5). The metabolism of arginine, proline, butanoate, and xenobiotic compounds by the cytochrome p450 pathway was markedly enriched in low-risk patients of both the training and validation cohorts. Some important energy metabolism pathways, such as glycolysis gluconeogenesis and amino-sugar and glutamate metabolism, were significantly enriched in the high-risk patients of the training cohort (Fig. 5A), and fatty acid and pyruvate metabolism pathways were dramatically enriched in the low-risk patients of the validation cohort (Fig. 5B). To further compare the key genes among different tumors, we conducted 
external validation using the Tumor IMmune Estimation Resource (TIMER) database (see Supplemental Figure S3). The expression profiles of these key genes were different in other tumors, suggesting heterogeneity between different tumors.

\section{Discussion}

Our work is the first step of establishing a metabolic prognostic model for GBM patients based on the GEO database, which included 18 metabolic genes affecting the prognosis of GBM patients. Independent external validation with the TCGA database proved that our model is able to effectively stratify and predict OS through the expression level of metabolic genes. In addition, our data suggest that the expression levels of COX10, COMT, and GPX2 were negatively related to OS, whereas the higher expression of OCRL and RRM2 were prone to a worse outcome. These findings indicate that the OS of GBM patients was significantly correlated with the expression levels of several metabolic genes, and the prognostic risk scores derived from our model are of great importance in predicting patient survival.

As the most common primary intracranial malignant tumor, the mortality rate of GBM remains high(5). Studies have proven that alterations in metabolic pathways are of great significance in tumor development(7), and metabolic genes are closely related to the prognosis of $\mathrm{GBM}(10)$. In recent years, predictive models of metabolic gene expression and mRNA characteristics have also become the focus of cancer prognosis(22). According to the heatmap of metabolic gene expression, no obvious difference was observed between the high- and low-risk groups at the individual gene level. Hence, it is hard to achieve an accurate conclusion for GBM prognosis prediction merely from a single gene analysis. Our research superiorly analyzed all differentially expressed metabolic genes and established a multiple gene expression model. In addition, due to the complexity of metabolic networks, a large number of metabolic genes are possibly involved in multiple pathways. For instance, GBE1 in our model is involved in galactose metabolism and glycogen metabolism, whereas EPHX1 plays a role in drug metabolism and naphthalene metabolism. Therefore, the multiple gene expression model is able to simulate the process of tumor metabolism more effectively and achieve better predictive potential.

Several studies have focused on prognostic models for GBM or glioma patients. Gittleman et al. constructed a nomogram using Cox proportional hazards regression of lower-grade glioma patients based on the TCGA database. In this model, tumor grade, age at diagnosis, and molecular subtype (IDH mutant with or without 1p/19q) were listed as independent risk factors, while sex and Karnofsky Performance Status (KPS) were statistically irrelevant to prognosis(23). Our metabolic prognostic model came to a similar conclusion that age at diagnosis and risk score were significantly related to OS. To reveal the relationship between the whole transcriptome and prognosis of GBM patients, Xu et al. performed weighted gene coexpression network analysis and constructed a four-gene prognostic model for GBM based on TCGA(24). Recently, metabolic alterations in glioma have become a research hotspot, and an energy metabolism-related signature with a Cox proportional hazards model for diffuse glioma was established by Zhou et al., by which patients could be distinguished with significantly different clinical and molecular features(25). Compared to prior methods, our model focused on all the metabolic 
genes enriched by KEGG pathways and applied Lasso-penalized Cox regression analysis, which is believed to be more accurate than stepwise selection(19). Nevertheless, molecular subtype and KPS were not included as risk factors in our model due to data limitations, which may have a certain impact.

The top 3 favorable genes that were positively related to OS were COX10, COMT and GPX2. COX10 encodes cytochrome $\mathrm{c}$ oxidase, which is the terminal component of the mitochondrial respiratory chain(26). Closely associated with mitochondrial oxidative phosphorylation, COX10 is involved in multiple tumors(27). Elevated COX10 expression levels in meningioma indicate higher aggressiveness and shorter progression-free survival(28); moreover, elevated COX10 levels are negatively correlated with the prognosis of glioma patients and may result in abnormal phosphorylation processes(29). We indeed observed a slightly increased expression of COX10 ( $\log _{2} F C=1.07$, see Supplemental table 52$)$, while our prognostic model suggested that it is a favorable gene in GBM, which is opposite to the findings of previous studies and deserves further investigation. COMT encodes catechol-0-methyltransferase, which catalyzes the transfer of a methyl group from S-adenosylmethionine to catecholamines, which is usually present in the metabolism of neurotransmitters and drugs(30). Studies have indicated that COMT can upregulate tumor suppressor genes by the PI3K/Akt pathway and inhibit the growth and invasion of cancer cells(31). COMT mutations are significantly correlated with cognitive impairment in brain tumor patients(32), and this conclusion is also applicable in glioma patients(33). Therefore, COMT may have a potential protective effect, and mutations in this gene may influence prognosis by affecting cognitive ability. Glutathione peroxidase 2 is encoded by GPX 2 and catalyzes the reduction in organic hydroperoxides and hydrogen peroxide, which thereby protects cells against oxidative damage(34). It has been reported that GPX2 is important in the progression of tumors and is negatively correlated with the prognosis of patients with $\mathrm{HCC}(35)$. However, the role of GPX2 in GBM has not been reported. Our model suggested that GPX2 was positively related to patient OS, indicating that protection against oxidative damage might exist in CNS tumors.

The unfavorable genes that indicate poor OS include OCRL and RRM2. OCRL encodes inositol polyphosphate 5-phosphatase and is mainly discussed in oculocerebrorenal syndrome of Lowe and Dent disease(36). Inositol polyphosphate 5-phosphatase regulates PI3K/Akt signaling, endocytosis, vesicle trafficking, and cell migration, proliferation and apoptosis(37). However, few studies have focused on the role and mechanism of OCRL in GBM, and more research on the relationship between them is needed. The protein encoded by RRM2 belongs to ribonucleotide reductase, which catalyzes the formation of deoxyribonucleotides from ribonucleotides. The high level of RRM2 expression has received extensive attention in several cancers(38). It has been proven that the overexpression of RRM2 can promote the proliferation, migration and invasion of GBM cells and inhibit apoptosis in cell experiments(39). The same results were observed in animal experiments, which may explain its influence on OS. The roles of these genes, especially OCRL and GPX2, in GBM remain far from distinct.

To further investigate the metabolic alterations in GBM patients, GSEA was conducted and revealed that differentially expressed metabolic genes were significantly enriched in certain signaling pathways. Hence, the molecules involved in these metabolic pathways may serve as diagnostic biomarkers and treatment 
targets. Our model is promising for reflecting the dysregulation of the metabolic microenvironment after further research and supporting metabolic therapy. According to published studies, most of these genes have been proven to be related to the occurrence and development of tumors. The genes are mainly involved in glucose metabolism, amino acid metabolism and DNA damage repair.

There are several limitations to this study. First, we included differentially expressed genes between GBM and normal brain tissues. Thus, some genes with promising influences on the prognosis of GBM were not included due to their nonsignificant expression differences. Second, there were several differences in patient populations between the GEO and TCGA datasets; therefore, bias in the data itself may introduce some disturbance to the model. Third, our prognostic model was based on separate metabolic genes, while the more complex mechanisms affecting the prognosis of GBM in the real world were not taken into consideration. In addition, GBM is known for its heterogeneous gene expression profiles, which obviously increases the difficulty in fitting the prognostic model. Our model would be strengthened by expanding the sample size, making adjustments and proving its effectiveness in a multicenter independent cohort. Additionally, subgroup analysis of the subtypes of GBM (proneural, neural, classical and mesenchymal) and IDH-related molecular classification are needed(40). Moreover, further basic studies to reveal the mechanism of relevant genes in the development and occurrence of GBM are vital.

\section{Conclusions}

In summary, our study developed a novel metabolic prognostic model for GBM based on the GEO dataset, which was validated by data from TCGA. Our model is capable of analyzing the risk level according to the expression of certain metabolic genes and predicting the survival of patients. In addition, the results reflected alterations in the metabolic microenvironment and indicated potential biomarkers for diagnosis and treatment.

\section{Declarations}

Ethics approval and consent to participate: Since this was a retrospective medical record review study of public database, written informed consent was waived.

Consent for publication: Not applicable

Availability of data and materials: The datasets used and/or analysed during the current study are available from the corresponding author on reasonable request.

Competing interests: The authors declare that they have no competing interests.

\section{Funding}

Yu Wang is supported by the Beijing Municipal Natural Science Foundation from the Beijing Municipal Science \& Technology Commission [grant number: 7202150]. Wenbin Ma is supported by the Beijing 
Municipal Natural Science Foundation from the Beijing Municipal Science \& Technology Commission [grant number: 19JCZDJC64200(Z)], the Chinese Academy of Medical Sciences Innovation Fund for Medical Sciences from the Chinese Academy of Medical Sciences [grant number: 2016-I2M-2-001], and the Tsinghua University-Peking Union Medical College Hospital Initiative Scientific Research Program from the Peking Union Medical College Hospital [grant number: 2019ZLH101].

\section{Authors' contributions}

Study design: LC, CW, MW, Yu Wang; Statistical Analysis: LC, CW; Manuscript writing: CW, LC, LP, KZ, Yaning Wang; Manuscript modification: DC, Yuekun Wang, MW, Yu Wang.

Acknowledgements: Not applicable

\section{References}

1.

Ostrom QT, Gittleman H, Truitt G, Boscia A, Kruchko C, Barnholtz-Sloan JS. CBTRUS Statistical Report: Primary Brain and Other Central Nervous System Tumors Diagnosed in the United States in 2011-2015. Neuro Oncol. 2018;20(suppl_4):iv1-86.

2.

Stupp R, Mason WP, van den Bent MJ, Weller M, Fisher B, Taphoorn MJ, et al. Radiotherapy plus concomitant and adjuvant temozolomide for glioblastoma. N Engl J Med. 2005;352(10):987-96. 3.

Mildenberger I, Bunse L, Ochs K, Platten M. The promises of immunotherapy in gliomas. Curr Opin Neurol. 2017;30(6):650-8.

4.

Behin A, Hoang-Xuan K, Carpentier AF, Delattre JY. Primary brain tumours in adults. Lancet. 2003;361(9354):323-31.

5 .

McNeill KA. Epidemiology of Brain Tumors. Neurologic clinics. 2016;34(4):981-98.

6.

Hanahan D, Weinberg RA. Hallmarks of cancer: the next generation. Cell. 2011;144(5):646-74.

7.

Petersen MC, Vatner DF, Shulman GI. Regulation of hepatic glucose metabolism in health and disease. Nat Rev Endocrinol. 2017;13(10):572-87.

8.

Cadoret A, Ovejero C, Terris B, Souil E, Levy L, Lamers WH, et al. New targets of beta-catenin signaling in the liver are involved in the glutamine metabolism. Oncogene. 2002;21(54):8293-301.

9 .

Leone RD, Zhao L, Englert JM, Sun IM, Oh MH, Sun IH, et al. Glutamine blockade induces divergent metabolic programs to overcome tumor immune evasion. Science (New York). 2019;366(6468):pp. 1013- 
10.

Yan H, Parsons DW, Jin G, McLendon R, Rasheed BA, Yuan W, et al. IDH1 and IDH2 mutations in gliomas. N Engl J Med. 2009;360(8):765-73.

11.

Agnihotri S, Zadeh G. Metabolic reprogramming in glioblastoma: the influence of cancer metabolism on epigenetics and unanswered questions. Neuro Oncol. 2016;18(2):160-72.

12.

Beiko J, Suki D, Hess KR, Fox BD, Cheung V, Cabral M, et al. IDH1 mutant malignant astrocytomas are more amenable to surgical resection and have a survival benefit associated with maximal surgical resection. Neuro Oncol. 2014;16(1):81-91.

13.

Toyonaga T, Yamaguchi S, Hirata K, Kobayashi K, Manabe O, Watanabe S, et al. Hypoxic glucose metabolism in glioblastoma as a potential prognostic factor. Eur J Nucl Med Mol Imaging. 2017;44(4):611-9.

14.

Feng L, Qian H, Yu X, Liu K, Xiao T, Zhang C, et al. Heterogeneity of tumor-infiltrating lymphocytes ascribed to local immune status rather than neoantigens by multi-omics analysis of glioblastoma multiforme. Scientific reports. 2017;7(1):6968.

15.

Yu X, Feng L, Liu D, Zhang L, Wu B, Jiang W, et al. Quantitative proteomics reveals the novel co-expression signatures in early brain development for prognosis of glioblastoma multiforme. Oncotarget. 2016;7(12):14161-71.

16.

Lee Y, Scheck AC, Cloughesy TF, Lai A, Dong J, Farooqi HK, et al. Gene expression analysis of glioblastomas identifies the major molecular basis for the prognostic benefit of younger age. BMC Med Genomics. 2008;1:52.

17.

Leek JT, Storey JD. Capturing heterogeneity in gene expression studies by surrogate variable analysis. PLoS Genet. 2007;3(9):1724-35.

18.

Diboun I, Wernisch L, Orengo CA, Koltzenburg M. Microarray analysis after RNA amplification can detect pronounced differences in gene expression using limma. BMC Genom. 2006;7:252.

19.

Tibshirani R. The lasso method for variable selection in the Cox model. Stat Med. 1997;16(4):385-95. 20.

lasonos A, Schrag D, Raj GV, Panageas KS. How to build and interpret a nomogram for cancer prognosis. J Clin Oncol. 2008;26(8):1364-70.

21. 
Subramanian A, Tamayo P, Mootha VK, Mukherjee S, Ebert BL, Gillette MA, et al. Gene set enrichment analysis: a knowledge-based approach for interpreting genome-wide expression profiles. Proc Natl Acad Sci U S A. 2005;102(43):15545-50.

22.

Liu GM, Xie WX, Zhang CY, Xu JW. Identification of a four-gene metabolic signature predicting overall survival for hepatocellular carcinoma. J Cell Physiol. 2020;235(2):1624-36.

23.

Gittleman H, Sloan AE, Barnholtz-Sloan JS. An independently validated survival nomogram for lower grade glioma. Neuro Oncol. 2019.

24.

Xu P, Yang J, Liu J, Yang X, Liao J, Yuan F, et al. Identification of glioblastoma gene prognosis modules based on weighted gene co-expression network analysis. BMC Med Genomics. 2018;11(1):96. 25.

Zhou Z, Huang R, Chai R, Zhou X, Hu Z, Wang W, et al. Identification of an energy metabolism-related signature associated with clinical prognosis in diffuse glioma. Aging. 2018;10(11):3185-209.

26.

Antonicka H, Leary SC, Guercin GH, Agar JN, Horvath R, Kennaway NG, et al. Mutations in COX10 result in a defect in mitochondrial heme $A$ biosynthesis and account for multiple, early-onset clinical phenotypes associated with isolated COX deficiency. Hum Mol Genet. 2003;12(20):2693-702.

27.

Chen Z, Li Y, Zhang H, Huang P, Luthra R. Hypoxia-regulated microRNA-210 modulates mitochondrial function and decreases ISCU and COX10 expression. Oncogene. 2010;29(30):4362-8.

28.

Schmidt M, Mock A, Jungk C, Sahm F, Ull AT, Warta R, et al. Transcriptomic analysis of aggressive meningiomas identifies PTTG1 and LEPR as prognostic biomarkers independent of WHO grade. Oncotarget. 2016;7(12):14551-68.

29.

Luan F, Chen W, Chen M, Yan J, Chen $\mathrm{H}, \mathrm{Yu} \mathrm{H}$, et al. An autophagy-related long non-coding RNA signature for glioma. FEBS Open Bio. 2019;9(4):653-67.

30 .

Lautala P, Ulmanen I, Taskinen J. Molecular mechanisms controlling the rate and specificity of catechol O-methylation by human soluble catechol O-methyltransferase. Mol Pharmacol. 2001;59(2):393-402. 31.

Wu W, Wu Q, Hong X, Xiong G, Xiao Y, Zhou J, et al. Catechol-O-methyltransferase inhibits colorectal cancer cell proliferation and invasion. Arch Med Res. 2015;46(1):17-23.

32.

Correa DD, Satagopan J, Cheung K, Arora AK, Kryza-Lacombe M, Xu Y, et al. COMT, BDNF, and DTNBP1 polymorphisms and cognitive functions in patients with brain tumors. Neuro Oncol. 2016;18(10):142533.

33. 
Altshuler DB, Wang L, Zhao L, Miklja Z, Linzey J, Brezzell A, et al. BDNF, COMT, and DRD2 polymorphisms and ability to return to work in adult patients with low- and high-grade glioma. Neurooncol Pract. 2019;6(5):375-85.

34.

Talmud PJ, Drenos F, Shah S, Shah T, Palmen J, Verzilli C, et al. Gene-centric association signals for lipids and apolipoproteins identified via the HumanCVD BeadChip. Am J Hum Genet. 2009;85(5):628-42. 35.

Liu T, Kan XF, Ma C, Chen LL, Cheng TT, Zou ZW, et al. GPX2 overexpression indicates poor prognosis in patients with hepatocellular carcinoma. Tumour Biol. 2017;39(6):1010428317700410.

36.

Hyvola N, Diao A, McKenzie E, Skippen A, Cockcroft S, Lowe M. Membrane targeting and activation of the Lowe syndrome protein OCRL1 by rab GTPases. Embo j. 2006;25(16):3750-61.

37.

Hakim S, Bertucci MC, Conduit SE, Vuong DL, Mitchell CA. Inositol polyphosphate phosphatases in human disease. Curr Top Microbiol Immunol. 2012;362:247-314.

38.

Furuta $\mathrm{E}$, Okuda $\mathrm{H}$, Kobayashi A, Watabe K. Metabolic genes in cancer: their roles in tumor progression and clinical implications. Biochim Biophys Acta. 2010;1805(2):141-52.

39.

Li C, Zheng J, Chen S, Huang B, Li G, Feng Z, et al. RRM2 promotes the progression of human glioblastoma. J Cell Physiol. 2018;233(10):6759-67.

40 .

Verhaak RG, Hoadley KA, Purdom E, Wang V, Qi Y, Wilkerson MD, et al. Integrated genomic analysis identifies clinically relevant subtypes of glioblastoma characterized by abnormalities in PDGFRA, IDH1, EGFR, and NF1. Cancer cell. 2010;17(1):98-110.

\section{Figures}




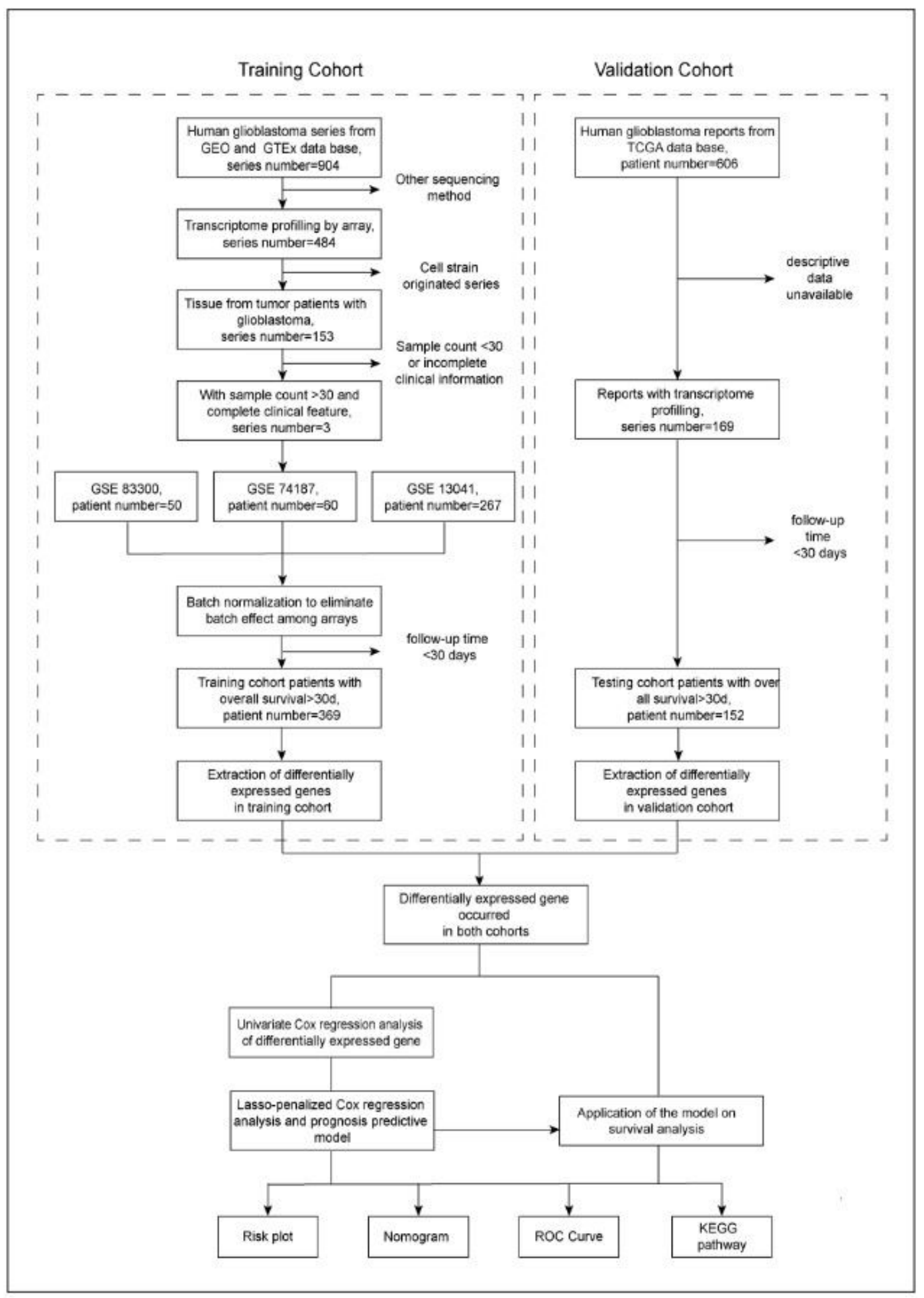

\section{Figure 1}

Flow chart presenting the whole design of the study. 
A

Risk + High risk + Low risk

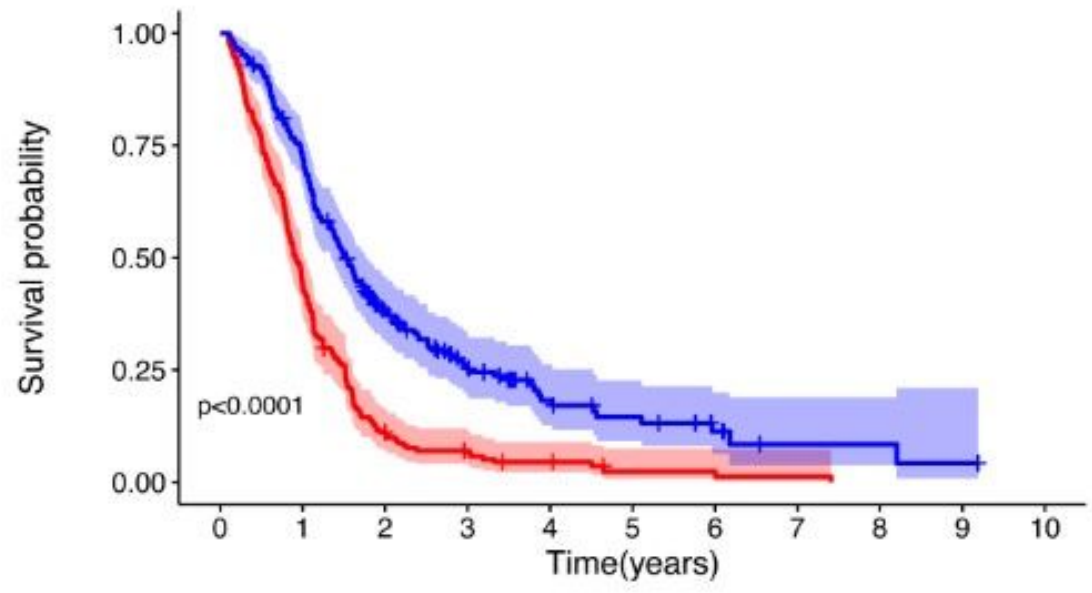

\begin{tabular}{|c|c|c|c|c|c|c|c|c|c|c|c|}
\hline \multirow[t]{2}{*}{ 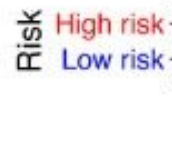 } & $\begin{array}{l}184 \\
185\end{array}$ & $\begin{array}{c}80 \\
132\end{array}$ & $\begin{array}{l}19 \\
60\end{array}$ & $\begin{array}{l}11 \\
32\end{array}$ & $\begin{array}{c}6 \\
16\end{array}$ & $\begin{array}{c}2 \\
11\end{array}$ & $\begin{array}{l}2 \\
6\end{array}$ & $\begin{array}{l}1 \\
2\end{array}$ & $\begin{array}{l}0 \\
2\end{array}$ & $\begin{array}{l}0 \\
1\end{array}$ & $\begin{array}{l}0 \\
0\end{array}$ \\
\hline & 0 & 1 & 2 & 3 & & 5 & 6 & 7 & 8 & 9 & 10 \\
\hline
\end{tabular}

B

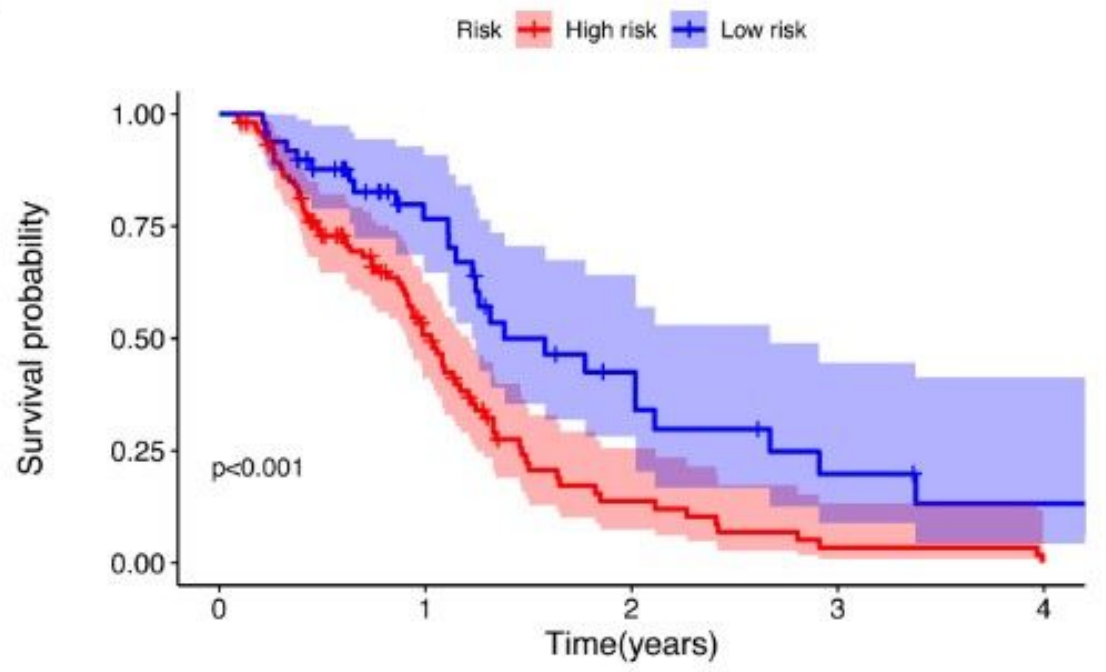

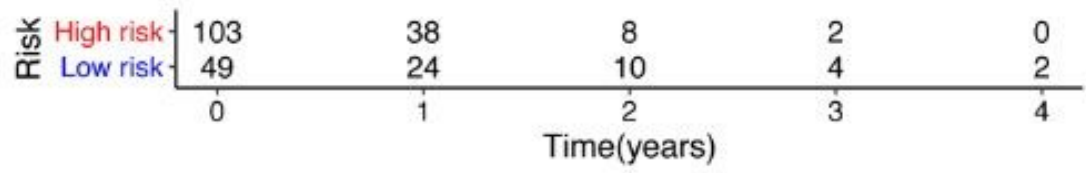

\section{Figure 2}

Kaplan-Meier survival curve for the training cohort $(A)$ and validation cohort (B). P value from the log-rank test. 
A

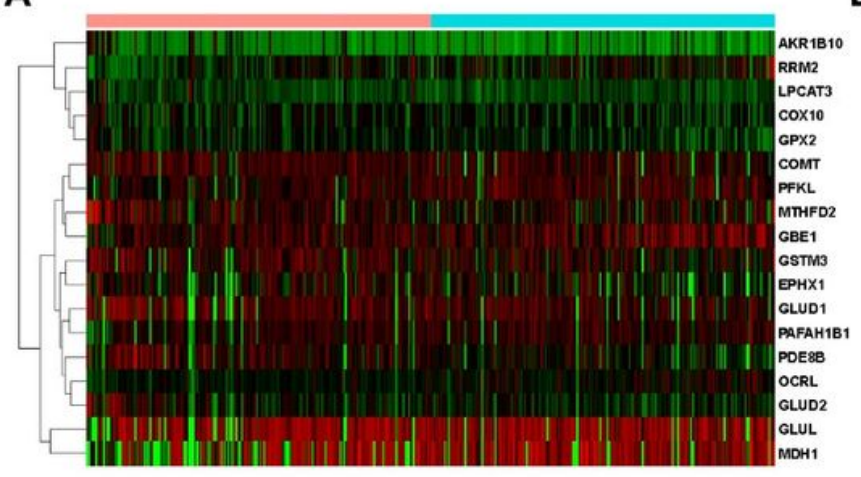

B
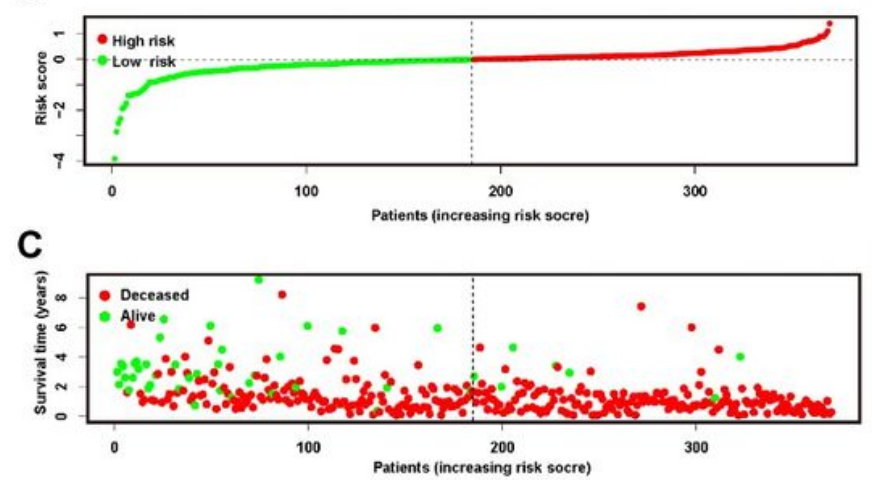

D

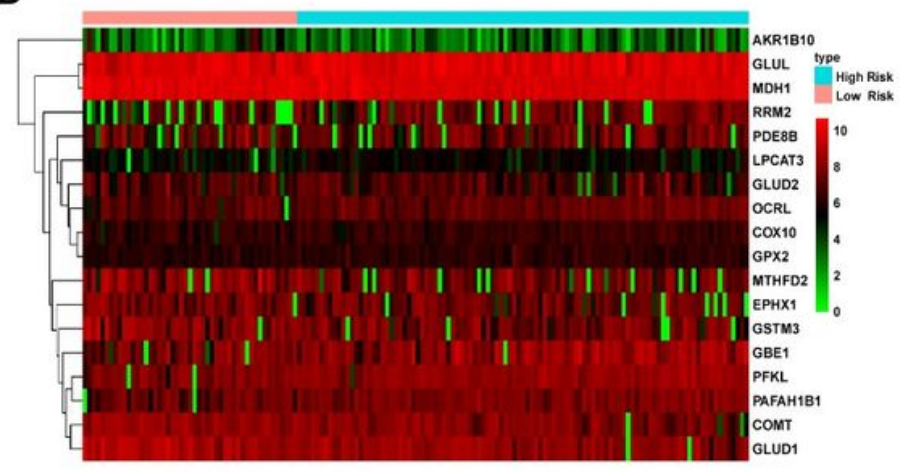

E

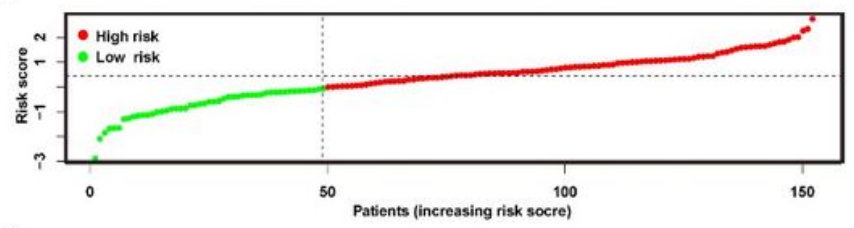

F

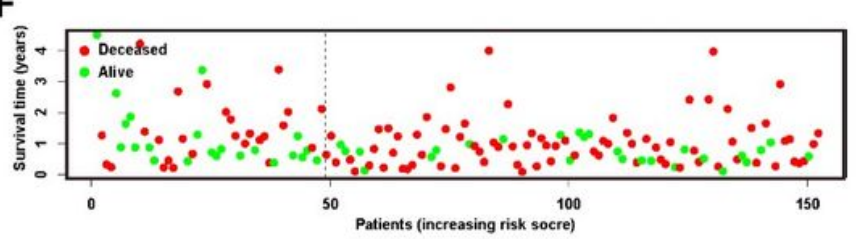

\section{Figure 3}

Risk plot of the training and validation cohorts. A heatmap of 18 metabolic genes showed the different expression patterns between high-risk and low-risk patients in the training (A) and validation (D) cohorts. (B) and (E) plotted the risk score of each patient and presented the cut-off value that defined high- and low-risk patients in the 2 cohorts, respectively. The OS of patients in the training $(\mathrm{C})$ and validation (F) cohorts were plotted according to the value of the risk score. OS: overall survival. 
A

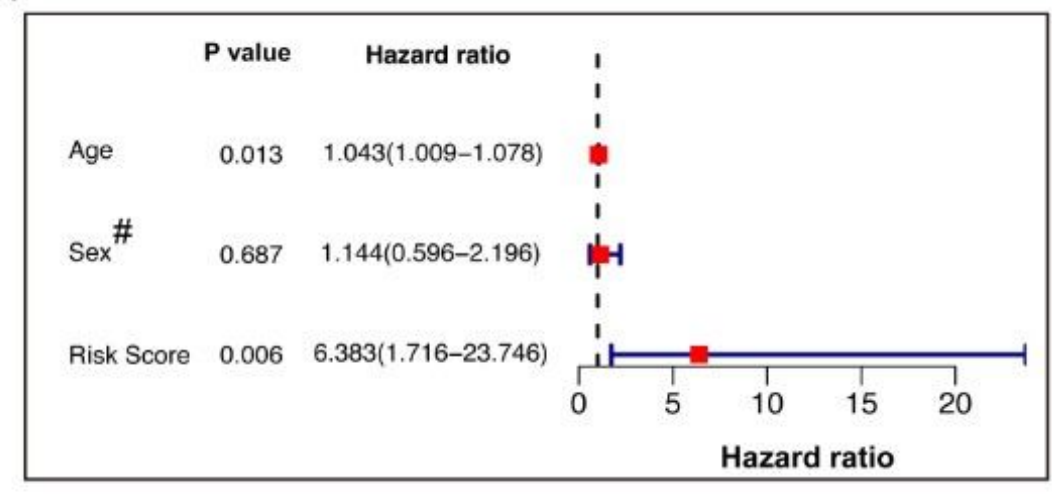

B

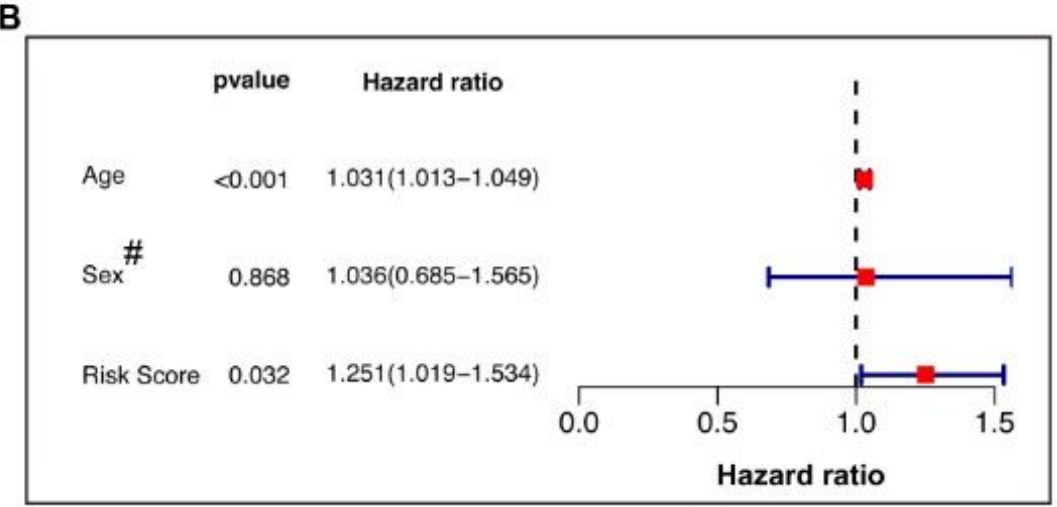

C

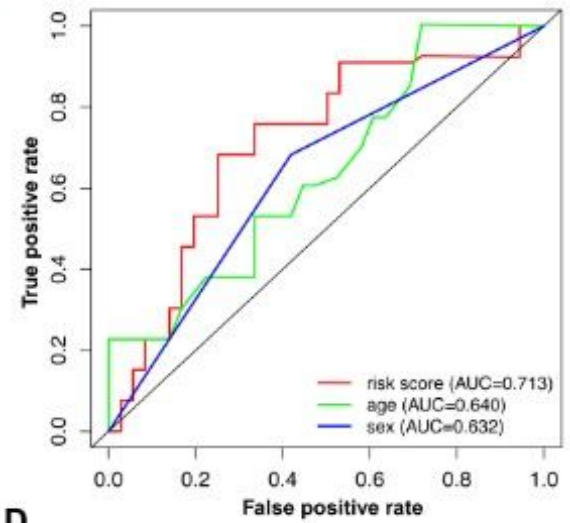

D

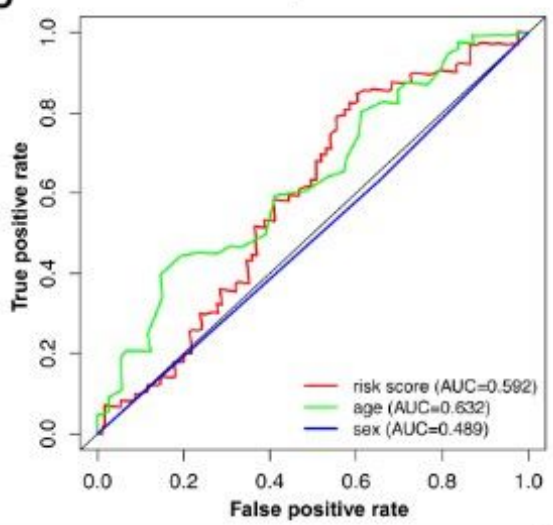

E

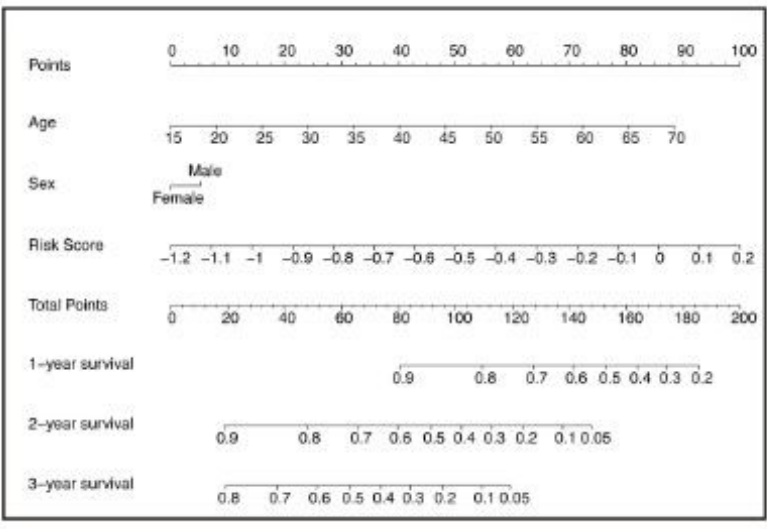

$F$

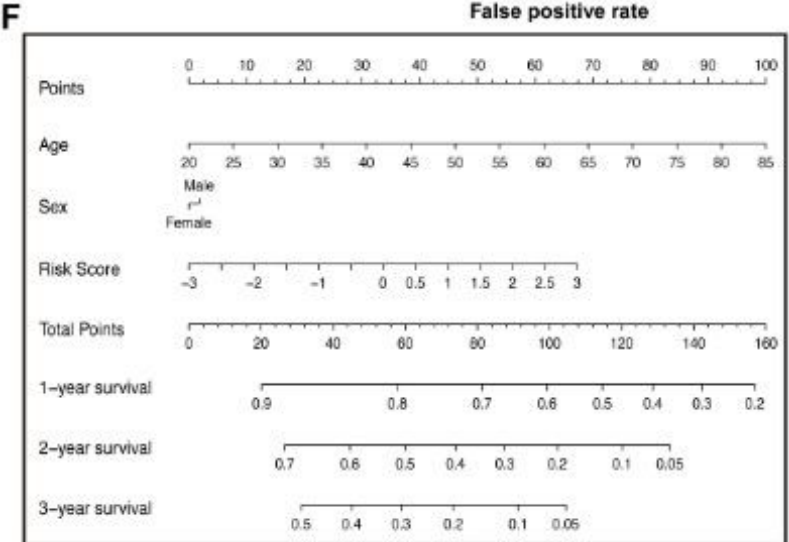

\section{Figure 4}

Cox regression, ROC analysis, and nomograms for patients in the training and validation cohorts. (A) Multivariate Cox regression of the training cohort. (B) Multivariate Cox regression of the validation cohort. The hazard ratios of age and risk score were 1.031 (95\% Cl 1.013-1.049) and 1.251 (95\% Cl 1.019-1.534), respectively, with $P$ values less than 0.05. (C) and (D) ROC analysis of age, sex, and risk score of patients in the training (C) and validation (D) cohorts. (E) and (F) Nomograms predicted the 1-, 2-, and 3-year survival of patients in the training $(E)$ and validation $(F)$ cohorts. \# Female=0; Male=1. ROC: receiver operating characteristic. 
A

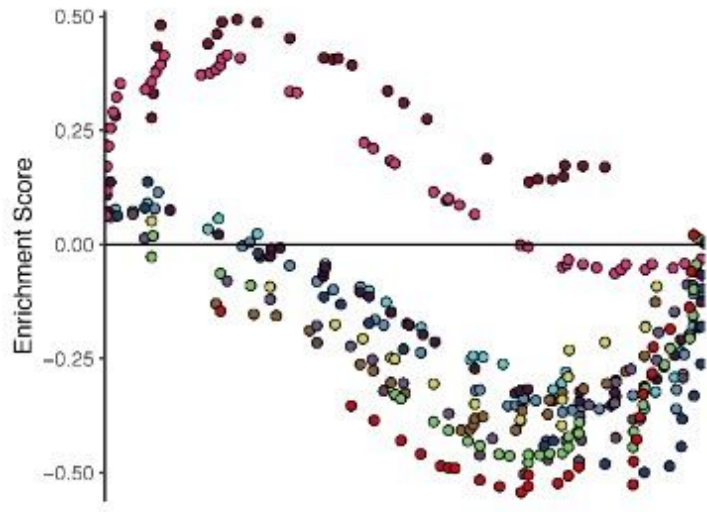

- Alanine aspartate and glutarnate metabolism

- Amino-sugar and nucleotide-sugar metabolism

- Arachidonic acid metabolism

- Arginine and proline metabolism

- Butanoate metabolism

- Glycine serine and threonine metabolism

- Glycolysis gluconeogenesis

- Histidine metabolism

- Linoleic acid metabolism

- Metabolism of xenobiotics by cytochrome p450

- Nitrogen metabolism

- Retinol metabolism

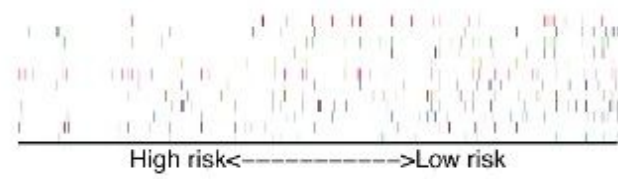

B

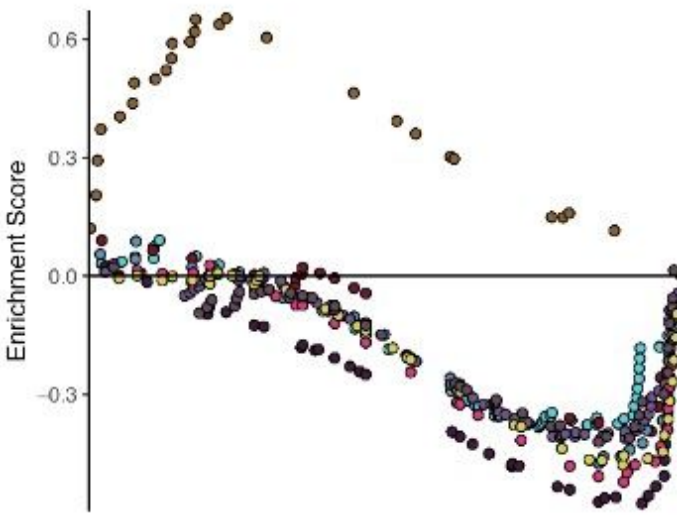

- Arginine and proline metabolism

- Beta alanine metabolism

- Butanoate metabolism

- Fatty acid metabolism

- Glycosaminoglycan biosynthesis heparan sulfate

- Metabolism of xenobiotics by cytochrome p450

- Propanoate metabolism

- Pyruvate metabolism

- Tryptophan metabolism

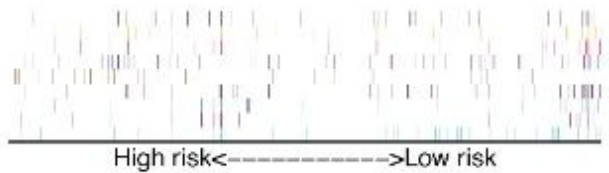

\section{Figure 5}

Multiple GSEA of the enriched KEGG pathways in the training (A) and validation (B) cohorts. GSEA: Gene set enrichment analysis.

\section{Supplementary Files}

This is a list of supplementary files associated with this preprint. Click to download.

- SupplementaltableS2.xls 
- SupplementalFigureS2.tif

- SupplementalFigureS3.tif

- SupplemetalTableS3.docx

- SupplementalFigures1.tif

- SupplementaltableS1.xls 